Editorial

\title{
A new approach to treatment vaccines preparation
}

\section{Editorial}

Therapeutic vaccines have been used for cancer immunotherapies more than decade. While these therapies have been with applied with some success until now we could not identify one therapeutic vaccine that could treat and/or prevent various cancers even one type of cancer in different patient.

Since the nature most malignant tumors is due to mutation of a single cell and each cell is different from others thus tumors in different patients would have not have same antigenic characteristics; hence, one cannot use a single therapeutic vaccine for all malignant tumors unless there are some homology in the tumor antigens and the vaccine we are using. This is the main reason for unsuccessful attempts of using current therapeutic vaccines in many patients.

In order to overcome tumor antigenic variation in therapeutic vaccines, I am proposing preparation of individual therapeutic vaccine. By this approach, each patient would have his or her own specific vaccine that would boost patient's immune system to eradicate cancer cells and/or prevent recurrence of the disease.

Preparation of very specific individual vaccine would be as follows: After surgery and removal of the tumor, the tumor lysate would be prepared and analyzed by proteomics procedure. Proteomics analysis with comparison to normal tissue lysate would identify protein(s) unique to the tumor. Next step is purification of the tumor specific protein(s). From this point one can use the purified protein as a therapeutic vaccine or sequence it for further characterization and perhaps making recombinant version of it for preparation of monoclonal antibody, which can be used for immunotherapy as well.
Volume I Issue 2 - 2015

Khansari $\mathrm{N}$

American Medical Diagnostic Laboratory, USA

Correspondence: Khansari N, Professor of Immunology, American Medical Diagnostic Laboratory, AMDL, 1665 Garden Grove Blvd, Garden Grove, CA 92843, Tel + I (949)228-8290, Emailnkhansari928@gmail.com

Received: October 23, 2015 | Published: October 27, 2015

\section{Acknowledgments}

None.

\section{Conflicts of interest}

Author declares there are no conflicts of interest.

\section{Funding}

None. 